\title{
Teaching Research on the Experimental Methods of Calculating the Charge-to-Mass Ratio of Electron
}

\author{
Xiaojuan Niu, Li Wang, Yafang Tu, Yu Tian \\ Department of Physics, School of Physics and Information Engineering \\ Jianghan University \\ Wuhan, China
}

\begin{abstract}
The charge-to-mass ratio of the electron is an important parameter in physics. There are many methods to calculate it, such as the Thompson method, Filter method, Magnetic focusing method, Zeeman Effect method, etc. This paper concentrates on the comparison and analysis of magnetic focusing method and Zeeman Effect method from three aspects of experimental principle, operation and relative error. Through in-depth analysis, the results show that the magnetic focusing method is suitable for students who are no-physics major. Compare with the Zeeman Effect method, the experimental principle and operation of magnetic focusing method are simpler. The experimental principle of Zeeman Effect method is relatively complex. It involves the knowledge of optics, electromagnetism and atomic physics. The Zeeman Effect method is more suitable for senior physics majors. The relative error of magnetic focusing method is $\mathbf{7 . 9 0 \%}$. The relative error of Zeeman method is smaller than the magnetic focusing method. It is $1.60 \%$.
\end{abstract}

Keywords-the charge-to-mass ratio of electron; Zeeman Effect; magnetic focusing method

\section{INTRODUCTION}

The ratio of the amount of charge and the mass of an electron $(e / m)$ is called the charge-to-mass ratio of an electron. The $e / m$ ratio is an important physical quantity to describe electron properties. In history, researchers firstly measured the charge-to-mass. Then they measured the amount of electron charge. Last, they got the mass of electron. This proves that atoms are separable[1]. The electron charge-to-mass ratio is most widely used in scientific fields of electron microscopy, cathode ray tubes, accelerator physics, atomic physics, Auger electron spectroscopy, cosmology and mass spectroscopy. The determination of this ratio is very important. In 1897, British physicist Thomson first calculated the charge-to-mass ratio. The method of Thomson was measured the $\mathrm{e} / \mathrm{m}$ ratio by means of opposing forces due to electric and magnetic fields on a beam of electrons from a cathode ray tube. He found that it was a fundamental particle having a negative charge e and a mass 2000 times, less than the lightest atom existed in all atoms. Thomson named this particle "corpuscle" but we now call it an electron. Because of the relativistic effect of mass, the charge-to-mass ratio of the electron decreases as the electron velocity increase. Therefore, now it is widely accepted the value for the charge-to-mass ratio of electron is $\mathrm{e} / \mathrm{m}=1.7588196 \times 10^{11} \mathrm{C} / \mathrm{kg}$. In most colleges and universities, the experiment to measure the electron charge to mass ratio is offering as a general physics experiment, modern physics experiment or electromagnetic experiment. The

This research was supported by National Natural Science Foundation of China (Grant No. 41104095) magnetic focusing method and the Zeeman Effect method are widely accepted. The following is a comparative analysis of the two methods from three aspects, to provide a comprehensive reference and selection for students in doing this experiment for different majors, as well as a good guide for students in doing this experiment, to achieve the purpose of high-quality teaching.

\section{COMPARISON AND ANALYSIS}

\section{A. Experimental principle}

1) The magnetic focusing method

The magnetic focusing method based on Thomson's idea. In an evacuated glass chamber (back-filled with low-pressure helium gas), electrons thermionic emitted from a heated metal filament are accelerated through a potential difference. The cyclotron motion of the resulting electron beam due to an applied magnetic field (from a Helmholtz coil apparatus) investigated to determine the ratio of the electrons' charge to their mass[2]. An electron of mass $m$ and charge $e$ released thermionic at the cathode has kinetic energy. This electron falls through the positive anode potential $U$. After accelerated by this high voltage electric field, the electron gaining kinetic energy of [3].

$$
\frac{1}{2} m v=e U
$$

The angle between the magnetic field of the Helmholtz coils and velocity is $\theta$, by the effect of diaphragm, this angle is small enough. The electronic speed decomposed into parallel with the component of the magnetic field $v_{\|}$and perpendicular to the magnetic field component $v_{\perp}$

$$
\left\{\begin{array}{l}
v_{\|}=v \cos \theta \approx v \\
v_{\perp}=v \sin \theta \approx v \theta
\end{array}\right.
$$

Perpendicular to the magnetic field, the electrons make circular motion with different radius $r$. Based on the second Newton law[5]:

$$
\mathrm{e} v_{\perp} B=\frac{m v_{\perp}^{2}}{r}
$$

The period of the electron in a circular motion is $\mathrm{T}$.

$$
\mathrm{T}=\frac{2 \pi r}{v_{\perp}}=\frac{2 \pi}{\frac{e}{m} B}
$$

Parallel to the magnetic field, the electrons move at a constant speed in a straight line. The parallel velocities of the electrons are approximately the same. The distance moved in a period is $h$. 


$$
\mathrm{h}=v_{\|} T=\frac{2 \pi v}{\frac{e}{m} B}
$$

Therefore, although the electrons go their separate ways at first, they come back together after a circle, which called a magnetic focusing. From this equation and Eq. (1), we obtain the expression for the charge-to-mass ratio of the electron.

$$
\frac{e}{m}=\frac{8 \pi^{2} U}{h^{2} B^{2}}
$$

Normally, the solenoid is a tightly wound in the experiment. We calculate magnetic field B using the Biot-Savart law.

$$
\mathrm{B}=\frac{\mu_{0} N I}{\sqrt{D^{2}+L^{2}}}
$$

Here $N$ is the number of turns of solenoid, $L$ is the length of the selenoid, $D$ is the diameter of the solenoid, $I$ is the focusing current, and the magnetic permeability constant is $\mu_{0}=4 \pi \times 10^{-7} \mathrm{H} / \mathrm{m}$.

Using Eq. (7) for the magnetic field B, we get

$$
\frac{e}{m}=\frac{8 \pi^{2}\left(L^{2}+D^{2}\right)}{\left(\mu_{0} N h\right)^{2}} \cdot \frac{U}{I^{2}}
$$

After the acceleration voltage given, the charge-to-mass ratio of the electron can obtained by measuring the focusing current $I$.

\section{2) The Zeeman Effect method}

In 1896, Pieter Zeeman discovered the influence of an external static magnetic field on the frequencies of spectral lines emitted by a light source, placed in that magnetic field. This phenomenon is known as the Zeeman Effect. If a spectral line splits into three lines, the splitting distance expressed by wave number is exactly equal to one Lorentz unit. This phenomenon called the Normal Zeeman effect. If one line splits more than three and the splitting distance is greater than or less than one Lorentz unit. This called the Abnormal Zeeman effect. The Zeeman Effect proves that the atomic has a magnetic moment and space quantization effect. The Zeeman Effect is one of the important methods to study the structure of atomic energy levels. The principle of Zeeman Effect experiment to calculate the electron charge-to-mass ratio is measurement the splitting distance of the spectral line in normal Zeeman Effect. The splitting distance is $L_{0}[4]$.

$$
L_{0}=\frac{e B}{4 \pi m c}
$$

According to the principle of normal Zeeman Effect, the spectral line split in magnetic field. Through Fabry-Perot interferometer and plus lens, these splitting lines from the interference rings at focal plane. The diameter $\mathrm{D}$ of the interference ring measured with a reading microscope. The difference of split wave's number is

$$
\tilde{v}=L_{0}=\frac{e B}{4 \pi m c}
$$

In terms of wavelength difference, then it is

$$
\Delta \lambda=\lambda^{2} \Delta \tilde{v}=\frac{\lambda^{2} e B}{4 \pi m c}
$$

Substitute the above equation into the formula of measuring wavelength difference, we get following equation.

$$
\frac{e}{m}=\frac{2 \pi c}{d B}\left(\frac{D_{1}^{2}-D_{2}^{2}}{D_{k-1}^{2}-D_{k}^{2}}\right)
$$

Here, $d$ is the spacing of Fabry-Perot interferometer. The external magnetic field measured by the tesla meter and the diameter of each ring measured from the photos of the Zeeman Effect splitting. The value of $\mathrm{e} / \mathrm{m}$ can be calculated by using Eq. (12).

Comparing the two experimental principles, the magnetic focusing method is mainly using the characteristics of the solenoid magnetic field and the law of motion charge in magnetic field. The experimental principle is relatively simple. This experimental method is suitable for non-physics majors' students who just studied the subject of college physics. The principle of the Zeeman Effect experiment is relativity complex and profound. It involves the knowledge of inner structures and spectral line of atoms, magnetic moment and angular momentum of atoms and Zeeman Effect. In addition, optical interference and Hall Effect are also involved in the experimental design. The experimenter needs to understand how the F-P interferometer works and how to measure the magnetic field by the principle of Hall Effect. Therefore, the Zeeman Effect is suitable for physics majors' students to choose. However, this experiment is very helpful to improve the comprehensive ability of students.

\section{B. Experiment operation}

The experimental operation of magnetic focusing method to measure electron charge-to-mass ratio is relatively simple. After connecting the circuit, it is only necessary to adjust the acceleration voltage to the given voltage value and observe the bright spot on the regulating fluorescent screen. Adjust the focus and brightness to bring the spot into focus. Then adjust the magnetization current from small to large, so that the light point on the fluorescent screen from point to spot, and then to point, record the voltage-current value when completing a focus. Plugin the formula to get the charge-to-mass ratio.

The experiment of measuring electron charge - mass ratio by Zeeman Effect is more complicated than that by magnetic focusing. In addition to the connected circuit, the experiment also involves the adjustment of the light path. The light beam needs to pass through the center of each optical element, and the parallelism of the F-P interferometer needs to adjust. The interference fringe is clear and does not change elliptically. After using CCD and computer to collect data, it is necessary to further measure the diameter of the ring generated by normal stuffing effect, and measure the magnetic induction intensity of the split magnetic field by using teslameter. Then we put the data into the formula to figure out the charge-to-mass ratio.

\section{Experimental results and error analysis}

The measurement data and calculation results of the two experimental methods given below. Table I shows the data of electron charge-mass ratio measured by magnetic focusing method. Table II shows the data of electron charge-mass ratio measured by Zeeman Effect method.

The electron charge-to-mass ratio is the average value of multiply measurements. Comparing the results of these two methods, the relative errors of the magnetic focusing method and the Zeeman Effect method respectively are 7.90\% and $1.60 \%$. The relative error of the Zeeman Effect method is obviously smaller than the magnetic focusing method. The 
reasons that causes error commonly have a geomagnetic field, the determination of the electron beam cross point start location, excitation current and pitch of spiral line [5]. To eliminate the effect of geomagnetic field, the solenoid can placed east-west or change the direction of the excitation current and take the average value of these two currents [6]. So the key to reducing the error is correctly determined by the excitation current value when the electron beam is focused. The ampere meter used in the experiment has a large measuring range. The small changes of current do not show on the ampere meter. This made excitation current value is not very precise. The relative error is large. By the Zeeman Effect method, the reason that cause error mainly has determination in the magnetic field during the experiment. Put the mercury lamp at the center of the magnetic field and the tesla meter at the center of the magnetic field when measuring the magnetic field. Make sure that the measured magnetic field is consistent with the magnetic field that splits the lines. The error of Zeeman Effect method is usually small. And Luo Jianfeng et al found the main reason accounting for big error is that the distance between the two pieces of reflecting mirror installed within Fabry-Perot standard device [7].

TABLE I. THE DATA OF ELECTRON CHARGE-MASS RATIO MEASURED BY MAGNETIC FOCUSING METHOD

HereN $=1385, \mathrm{~L}=265, \mathrm{D}=92.5 \mathrm{~mm}, \mathrm{~h}=201 \mathrm{~mm}$.
\begin{tabular}{|c|c|c|c|c|c|c|}
\hline$U$ & $750 \mathrm{v}$ & $800 \mathrm{v}$ & $850 \mathrm{v}$ & $900 \mathrm{v}$ & $950 \mathrm{v}$ & $1000 \mathrm{v}$ \\
\hline$+I$ & 0.467 & 0.497 & 0.529 & 0.557 & 0.605 & 0.654 \\
\hline$-I$ & 0.457 & 0.489 & 0.524 & 0.548 & 0.586 & 0.621 \\
\hline $\bar{I}$ & 0.462 & 0.493 & 0.527 & 0.553 & 0.596 & 0.638 \\
\hline & & & & & & \\
$\frac{U}{I^{2}}\left(\times 10^{3}\right)$ & 4.686 & 3.292 & 3.060 & 2.943 & 2.675 & 2.457 \\
\hline$e / m\left(\times 10^{11}\right)$ & 2.382 & 1.674 & 1.556 & 1.496 & 1.360 & 1.249 \\
\hline$\overline{e / m}\left(\times 10^{11}\right)$ & & & & & & \\
\hline Relative error & \multicolumn{7}{|c|}{$7.620 \%$} \\
\hline
\end{tabular}

TABLE II. THE DATA OF ELECTRON CHARGE-MASS RATIO MEASURED BY ZEEMAN EFFECT METHOD.

\begin{tabular}{|c|c|c|c|c|c|}
\hline Times & 1 & 2 & 3 & 4 & 5 \\
\hline$e / m$ & 1.698 & 1.735 & 1.710 & 1.740 & 1.770 \\
\hline$\overline{e / m}$ & \multicolumn{5}{|c|}{1.731} \\
\hline Relative error & \multicolumn{5}{|c|}{$1.60 \%$} \\
\hline
\end{tabular}

\section{CONCLUSION AND DISCUSSION}

The purpose of setting up physics experiment is to let students master how to observe physical phenomena and study physical laws with experiment. Through experimental teaching, and let students understand the content involved in the experiment and its application in practice. By visual experimental phenomena, students' vision can be broadened. Through experimental operation and data analysis, students' manipulative skills and comprehensive design ability can be improved. It is very important to arouse students' learning enthusiasm. Both experiments of magnetic focusing and the Zeeman Effect are all calculated the charge-to-mass ratio of electron. However, their experimental principles are different. Therefore, the requirement of experimenters is different. The magnetic focusing method is relatively simple and suitable for non-physical major students who have taken college physics courses. Through this experiment, let the experimenter master the principle of magnetic focusing and understand the force and motion characteristics of moving charge in magnetic and electric fields. Nevertheless, the experimental principle of Zeeman Effect is quite complicated. It is a comprehensive experiment. This method is suitable for physics major students who have studied general physics, atomic physics and quantum mechanics. In addition, the operation method of this experiment is relatively complex. Through this experiment, students not only master the basic experimental principles but also exercise the hands-on ability and experimental comprehensive design ability. Comparing the results of these two methods in actual teaching, the error of the Zeeman Effect method is smaller than the magnetic focusing.

\section{REFERENCES}

[1] L. Jinbo and G. Hailin, "Determination of the starting point of electron beam helix in electron charge-to-mass ration experiment by magnetic focusing method,” J. Nanyang Normal University, Vol.9, pp.37-38, September. 2009. (In Chinese)

[2] Y.K. Yang, "Determining the ratio of chare to mass e/m for electrons by magnetic focusing”, Amer. J. Phys., Vol. 66, pp. 157-162, 1998.

[3] L. Canbin, Q. Guangrong and L. Zhujian, "Electromagnetics, "Higher education press, pp. 193-195, December. 2010. (In Chinese)

[4] Z. Lihui, N. Xiaojuan and T. Yu, Modern Physics experiments, Science press, pp. 99-110, September. 2017. (In Chinese)

[5] W. Qi, L. Dianqing and L. Xinhua, "Experimental derive of electron beam measuring the charge-mass ratio of electron and analysis on the uncertainty of result surveyed”, Expe. Tech. Mana., vol. 23, pp. 26-28, February. 2006. ( In Chinese)

[6] Y. Shehui and T. Rui, "Analysis of magnetic focusing method to electron charge-mass ratio experiment ", experiment science and technology, vol. 12, pp. 1-3, February. 2014. (In Chinese)

[7] L. Jiangfeng, L. Yong, K. Dongli and Y. Hongwei, "Examining and analyzing for the measurement error of charge-mass ratio of electron by Zeeman effect experiment”, Phys. Exper. Coll., Vol. 24. pp. 75-78, August 2011. (In Chinese) 